\title{
Impact of pre-enrolment medication use on clinical outcomes in SUMMIT
}

\author{
Jørgen Vestbo ${ }^{1,2}$, Mark Dransfield ${ }^{3}$, Julie A. Anderson ${ }^{4}$, Robert D. Brook ${ }^{5}$, \\ Peter M.A. Calverley ${ }^{6}$, Bartolome R. Celli $\mathbb{1}^{7}$, Nicholas J. Cowans ${ }^{8}$, \\ Courtney Crim ${ }^{9}$, Fernando Martinez ${ }^{5,10}$, David E. Newby ${ }^{11}$, Julie Yates ${ }^{9}$ and \\ Peter Lange ${ }^{12,13}$, on behalf of the SUMMIT Investigators
}

Affiliations: ${ }^{1}$ Division of Infection, Immunity and Respiratory Medicine, School of Biological Sciences, The University of Manchester, Manchester, UK. ${ }^{2}$ North West Lung Centre, Manchester University NHS Foundation Trust, Manchester, UK. ${ }^{3}$ Lung Health Center, University of Alabama at Birmingham, Birmingham, AL, USA ${ }^{4}$ Research and Development, GlaxoSmithKline, Uxbridge, UK. ${ }^{5}$ University of Michigan Health System, Ann Arbor, MI, USA. 'University of Liverpool, Dept of Medicine, Clinical Sciences Centre, University Hospital Aintree, Liverpool, UK. ${ }^{7}$ Pulmonary and Critical Care Division, Brigham and Women's Hospital, Harvard Medical School, Boston, MA, USA. ${ }^{8}$ Veramed Ltd, Twickenham, UK. ${ }^{9}$ Research and Development, GlaxoSmithKline, Research Triangle Park, NC, USA. ${ }^{10}$ Division of Pulmonary and Critical Care Medicine, Weill Cornell Medicine, New York, NY, USA. ${ }^{11}$ British Heart Foundation Centre for Cardiovascular Science, University of Edinburgh, Edinburgh, UK. ${ }^{12}$ Dept of Public Health, Section of Social Medicine, University of Copenhagen, Copenhagen, Denmark.

${ }^{13}$ Medical Unit, Respiratory Section, Herlev Hospital, Copenhagen University Hospital, Copenhagen, Denmark.

Correspondence: Jørgen Vestbo, 2nd Floor, ERC Building, Wythenshawe Hospital, Southmoor Road, Manchester, M23 9LT, UK. E-mail: jorgen.vestbodmanchester.ac.uk

ABSTRACT The impact of prior treatment on results of clinical trials in chronic obstructive pulmonary disease (COPD) has been debated. We used data from the Study to Understand Mortality and Morbidity in COPD Trial to examine the impact of prior treatment on the effects of randomised study drugs on mortality and exacerbations.

We used data on 16417 patients with moderate COPD and heightened cardiovascular risk and information on prior medications to examine the effects of fluticasone furoate (FF), vilanterol (VI) and combined FF/VI compared to placebo on moderate and severe exacerbation as well as mortality. The study was event-driven with a median study exposure of 1.8 years. This study was registered with ClinicalTrials.gov, number NCT01313676.

There were no consistent associations between treatment prior to study entry and the effects of FF, VI or FF/VI on exacerbations during the study. However, patients taking inhaled corticosteroids and one or more bronchodilators prior to study entry seemed to have a better effect of active treatments than of placebo on mortality (hazard ratio for FF/VI 0.65 , 95\% CI $0.48-0.89$ ). Survival in those randomised to placebo was independent of treatment prior to study enrolment.

Prior treatment appears to affect treatment effects on mortality but not exacerbations in a randomised controlled trial of patients with COPD and heightened cardiovascular risk.

@ERSpublications

In mortality trials including patients with COPD and heightened cardiovascular risk, patients' treatments before entering the trial may affect the effects of inhaled medications during the study http://ow.ly/WRRb30nwyIE

Cite this article as: Vestbo J, Dransfield M, Anderson JA, et al. Impact of pre-enrolment medication use on clinical outcomes in SUMMIT. ERJ Open Res 2019; 5: 00203-2018 [https://doi.org/10.1183/ 23120541.00203-2018].

This article has supplementary material available from openres.ersjournals.com

Received: Oct 302018 | Accepted after revision: Dec 192018

This study is registered at www.clinicaltrials.gov with identifier number NCT01313676. GSK is committed to full data sharing through the portal https://www.clinicalstudydatarequest.com.

Copyright @ERS 2019. This article is open access and distributed under the terms of the Creative Commons Attribution Non-Commercial Licence 4.0. 


\section{Introduction}

Clinical trials in chronic obstructive pulmonary disease (COPD) often require recruited patients to discontinue their usual inhaled respiratory medications before they are randomised to study interventions [1-4]. As these trials also require patients to be clinically stable at the time of their enrolment, this approach leads to the withdrawal of what is effective treatment for many patients. This can potentially lead to a withdrawal effect, where events are seen soon after stopping a treatment and entering a trial. However, there can be later and more subtle effects of changing treatment as part of entering a trial. Subsequent randomisation to a new regimen may be superior to the previous treatment, similarly effective or less effective. The latter may occur when patients are randomised from active treatment to a placebo or an inferior treatment regime. This may impact events in the longer term; for example, affecting exacerbation rates subsequent to study entry. Although the methodology of discontinuation of existing medication is common, it is not the same as recruiting a treatment-naïve population in which all randomised patients are started on new therapy where there is no potential for a withdrawal effect. Thus, recruitment of patients on maintenance therapies can impact individual patient safety and outcomes as well as the interpretation of trials as a whole [5].

The potential for this approach to bias the results of large clinical trials has been discussed most frequently for studies of inhaled corticosteroids (ICS) with exacerbations as the outcome [5-7]. In these trials, patients already taking ICS were randomised either to continue to take them or to have them withdrawn while those who were ICS naïve were randomised to start them de novo or to start a placebo. When the effect of ICS on exacerbation risk was examined separately in the ICS pre-treated and ICS naïve groups in the OPTIMAL trial, the benefit of randomisation to ICS was only apparent in the pre-treated group [5]. In contrast, an analysis of the TORCH study showed that ICS and ICS/long-acting $\beta_{2}$-agonists (LABA) (as well as LABA alone) reduced the risk of exacerbations compared with placebo regardless of whether patients were taking ICS prior to randomisation [7]. This TORCH analysis also showed a reduction in exacerbations with active treatments regardless of prior LABA use, but there are no other published data about the impact of prior long-acting muscarinic antagonist or LABA use on outcomes.

The recently published Study to Understand Mortality and Morbidity in COPD Trial (SUMMIT) is one of the largest COPD studies ever conducted, randomising $>16000$ patients with heightened cardiovascular risk to ICS alone (fluticasone furoate (FF)), LABA alone (vilanterol (VI)), combination ICS/LABA (FF/VI), or matched placebo to compare survival [2]. The trial provides a unique opportunity to examine the impact of prior medication use on the mortality and risk of exacerbations after randomisation, as well as study the safety issues around entering previously treated patients with moderate COPD into a placebo-controlled trial. These analyses are all post hoc subgroup analyses that should be interpreted with caution.

\section{Methods}

\section{Study design and patients}

Details of the study have been published previously $[2,8]$. Briefly, this was a prospective, double-blind, parallel-group, placebo-controlled, event-driven, randomised trial conducted at 1368 centres in 43 countries. Participants had moderate COPD according to the Global Initiative for Chronic Obstructive Lung Disease and heightened cardiovascular risk. Participants were allocated equally to one of four

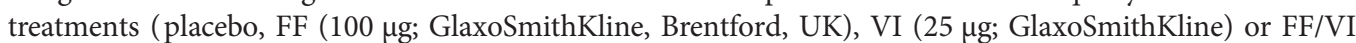
(100/25 $\mu$ g; Relvar/Breo, GlaxoSmithKline)) administered once daily as a dry powder with the use of an inhaler (Ellipta, GlaxoSmithKline). The primary outcome was mortality, and the secondary outcomes were on-treatment rates of decline in forced expiratory volume in $1 \mathrm{~s}$ (FEV1), and the on-treatment composite cardiovascular endpoint of cardiovascular death, myocardial infarction, stroke, unstable angina and transient ischaemic attack. All patients provided written informed consent. The study was approved by local ethics committees and was conducted in accordance with the Declaration of Helsinki and Good Clinical Practice guidelines. The study is registered at www.clinicaltrials.gov with identifier number NCT01313676.

\section{Outcome measurements and analyses}

For these analyses, we examined mortality and exacerbations. As this was an event-driven study in which follow-up continued until $\geqslant 1000$ deaths had occurred, survival status was assessed by all study sites at the "common end date", January 25, 2015. The median study exposure was 1.8 years.

To evaluate the effect of prior medication on treatment estimates during the study, we analysed the population as they were randomised. Mortality was analysed using a Cox proportional hazards model allowing for covariates of age and sex. Exacerbations were assessed every 3 months and at the end of study treatment. Time to first exacerbation was analysed using a Cox proportional hazards model allowing for covariates of age, sex and exacerbations in the 12 months prior to study entry. Moderate exacerbations 
were defined as a symptomatic deterioration requiring treatment with antibiotic agents and/or systemic corticosteroids, whereas severe exacerbations of COPD were those leading to hospital admission. For these analyses, the reduction in exacerbation risk was expressed as a percentage derived from the hazards ratio of the analysis of time to first exacerbation. Additional post hoc analyses were performed to investigate the effect of withdrawal of medications prior to the study. Prior therapy was defined as taking a long-term COPD medication ( $\geqslant 30$ days) and stopping this medication prior to enrolment. Subjects within each treatment arm were categorised to one of four groups: not withdrawn from inhaled maintenance COPD medication (none); withdrawn from ICS alone; withdrawn from a long-acting bronchodilator (LABD) alone; or withdrawn from a combination of ICS and a LABD, most often a fixed ICS/LABA combination. As few patients were previously on ICS alone or on only LABD, we report these analyses in the supplementary material only.

To evaluate safety, we also analysed the time to death in subjects on placebo to assess the effect of baseline treatment. As covariates in the analysis, we included age, sex, baseline FEV1, cardiovascular risk, history of exacerbations, race, region, smoking status at screening, and indicators of ischaemic and vascular disease.

\section{Results}

Of the 16485 patients included in the intention-to-treat analysis, we had information on previous use of inhaled medications in 16417. At study entry, 9423 (57\%) were treatment naïve regarding inhaled COPD maintenance treatment, 643 (4\%) were on an ICS alone, 1734 (11\%) were on an inhaled LABD alone, and 4617 (28\%) were on a combination of ICS and one or more LABD. Apart from those on ICS before entering the study having a higher proportion of former smokers and exacerbators, there were no differences between the groups (table 1). As expected, these four subgroups of previous treatment were distributed similarly between the four study treatment groups (placebo, FF, VI and FF/VI) as a result of the large study population.

\section{Outcomes according to prior treatment}

As previously reported, FF/VI did not reduce overall mortality compared with placebo: relative reduction $12 \%$ (95\% CI -4-26\%) [2]. However, variations existed when data were split into subgroups. When analysing the effect of randomised treatment on mortality for each of the pre-treatment groups, the treatment effect varied widely (figure 1 and figure S1). Patients previously on both ICS and one or more LABD seemed to have a better effect from using any study treatment (FF, VI or FF/VI) compared with placebo, with the difference being greatest in those randomised to FF/VI where there was a 35\% (95\% CI $11-52 \%)$ lower risk of mortality than those randomised to placebo (figure 1b). The corresponding reductions were $24 \%(95 \% \mathrm{CI}-3-44 \%)$ in patients randomised to $\mathrm{FF}$ and $19 \%$ (95\% CI $-9-40 \%)$ in those

\section{TABLE 1 Baseline demographics according to previous treatment}

\begin{tabular}{|c|c|c|c|c|}
\hline & No previous COPD medication & Previous ICS only & Previous LABD only & Previous ICS/LABD \\
\hline Subjects & 9423 & 643 & 1734 & 4617 \\
\hline Age years & 64.7 & 64.3 & 66.1 & 66.0 \\
\hline Females & $2325(25 \%)$ & $201(31 \%)$ & $429(25 \%)$ & $1231(27 \%)$ \\
\hline Former smokers & $4562(48 \%)$ & $383(60 \%)$ & $920(53 \%)$ & 2900 (63\%) \\
\hline Smoking pack-years & 40.0 & 37.8 & 43.6 & 41.5 \\
\hline \multicolumn{5}{|l|}{ Region } \\
\hline USA & 1469 (16\%) & $63(10 \%)$ & $218(13 \%)$ & $803(17 \%)$ \\
\hline Asia ${ }^{\#}$ & $1961(21 \%)$ & $35(5 \%)$ & $121(7 \%)$ & $561(12 \%)$ \\
\hline Europe $1^{\Uparrow}$ & $2139(23 \%)$ & $88(14 \%)$ & $1175(68 \%)$ & $1872(41 \%)$ \\
\hline Europe $2^{+}$ & 3076 (33\%) & $352(55 \%)$ & $172(10 \%)$ & $1025(22 \%)$ \\
\hline RoW ${ }^{\S}$ & $778(8 \%)$ & $105(16 \%)$ & $48(3 \%)$ & $356(8 \%)$ \\
\hline
\end{tabular}

Data are presented as mean, unless otherwise stated. COPD: chronic obstructive pulmonary disease; ICS: inhaled corticosteroids; LABD: long-acting bronchodilators; BMI: body mass index; FEV1: forced expiratory volume in $1 \mathrm{~s}$; RoW: rest of world. \#: China, Indonesia, India, Japan, Korea, Malaysia, Philippines, Taiwan, Thailand and Vietnam; ๆ: Austria, Belgium, Bulgaria, Czech Republic, France, Germany, Greece, Hungary, Latvia, Netherlands, Poland, Romania, Slovakia, Spain, UK and Croatia; ${ }^{+}$: Bosnia and Herzegovina, Belarus, Georgia, Israel, Macedonia (Former Yugoslav Republic of), Russian Federation, Serbia, Turkey and Ukraine; ${ }^{\S}$ : Argentina, Australia, Canada, Chile, Columbia, Mexico and South Africa. 

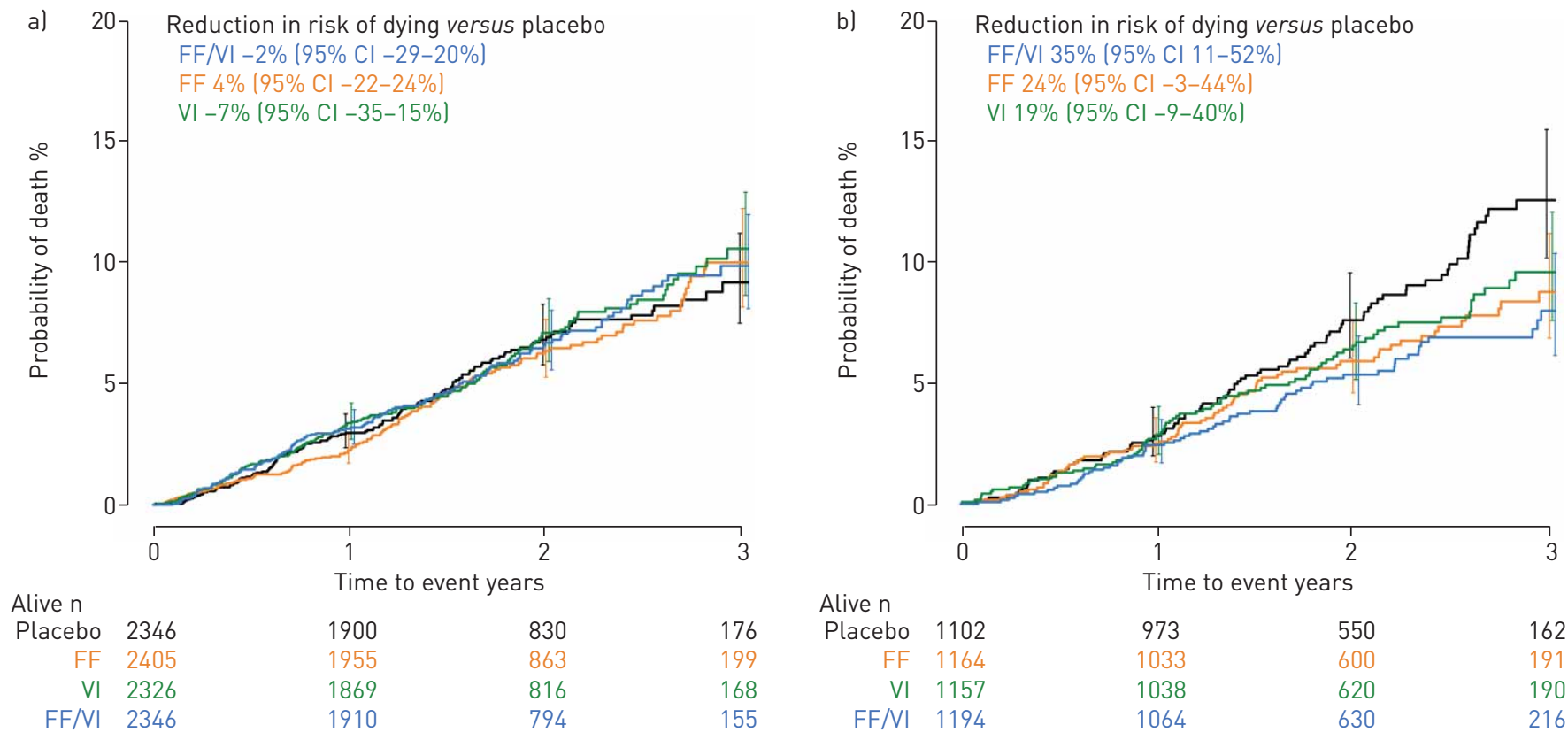

FIGURE 1 Overall mortality in the four treatment groups (placebo, fluticasone furoate (FF), vilanterol (VI) and FF/VI) according to previous treatment use. a) Those receiving no inhaled pre-treatment maintenance medication; b) those on inhaled corticosteroids and long-acting bronchodilators as previous treatment use.

randomised to VI. For those previously on either ICS alone or a LABD alone, there was no clear pattern (figure S1). We performed a global test of interaction for the medications on mortality: $\mathrm{p}=0.440$.

We subsequently analysed time to first moderate or severe COPD exacerbation. In the overall study, all three treatments reduced risk of exacerbations, by $10 \%$ (95\% CI 2-18\%), 12\% (95\% CI 4-19\%) and $29 \%$ (95\% CI 22-35\%) for the VI, FF and FF/VI groups, respectively. As shown in table 2, figure 2 and figure S2, the reductions in risk of exacerbations varied considerably, as illustrated by the wide $95 \%$ confidence intervals, but there were no consistent differences from the overall study findings. The global test of interaction for the medications on exacerbations resulted in a p-value of 0.450 .

\section{Safety of being allocated to placebo}

Although patients in SUMMIT were randomly allocated to placebo, FF, VI or FF/VI, and therefore have similar study exposure, this is not the case when we look at groups defined according to prior treatment. Study exposure for treatment-naïve patients was 1.77 years, for those having had ICS alone 2.15 years, LABD alone 2.08 years and prior combination treatment 2.06 years, highlighting the need for a survival analysis rather than simply comparing the percentage of deaths in each group. In the analysis within the group randomised to placebo, we found no effect of prior treatment on mortality. The hazard ratio for prior ICS alone versus no prior treatment was 0.97 (95\% CI $0.53-1.78$ ), for prior LABD alone versus no prior treatment 0.79 (95\% CI $0.52-1.21)$ and for combination treatment versus no prior treatment 1.08 (95\% CI 0.82-1.42)

\section{Discussion}

This is the largest study examining impact of prior use of inhaled COPD maintenance treatment on important outcomes in a randomised placebo-controlled trial. Whereas most previous analyses have focused on exacerbations only, we have, in addition, included overall mortality. Our study showed that having received ICS and/or LABD prior to study entry appeared to affect treatment effects on mortality whereas it did not seem to have a systematic bias on treatment effects regarding risk of exacerbations. It should be noted that these analyses are all post hoc subgroup analyses that should be interpreted with caution.

Patients on treatment before entering a trial will be different from those not receiving any prior treatment; i.e. there is a reason for them being on medication. In SUMMIT, this was apparent in the group with ICS as previous treatment use: they had a history of more frequent exacerbations, which is consistent with ICS being recommended for reducing the risk of exacerbations and in keeping with reports from other large studies [9]. It is unclear if these features in themselves could affect the results of a trial. Rates of exacerbations will almost invariably be higher in all groups with prior use than in treatment-naïve 


\begin{tabular}{|c|c|c|c|c|}
\hline & Placebo & FF $100 \mu \mathrm{g}$ & VI $25 \mu g$ & FF/VI 100/25 $\mu \mathrm{g}$ \\
\hline Total subjects & 4111 & 4135 & 4118 & 4121 \\
\hline \multicolumn{5}{|c|}{ Subjects who did not receive LABD prior to the study } \\
\hline \multicolumn{5}{|l|}{ None } \\
\hline Hazard ratio $(95 \% \mathrm{CI})$ & & $0.952(0.852-1.065)$ & $0.844(0.752-0.947)$ & $0.801(0.713-0.900)$ \\
\hline Reduction in risk \% $(95 \% \mathrm{CI})$ & & $4.8(-6.5-14.8)$ & $15.6(5.3-24.8)$ & $19.9(10.0-28.7)$ \\
\hline \multicolumn{5}{|l|}{ ICS alone } \\
\hline Subjects & 176 & 148 & 169 & 150 \\
\hline \multicolumn{5}{|l|}{ Active versus placebo } \\
\hline Hazard ratio $(95 \% \mathrm{Cl})$ & & $0.889(0.588-1.344)$ & $1.180(0.811-1.717)$ & $0.877(0.582-1.322)$ \\
\hline Hazard ratio $(95 \% \mathrm{CI})$ & & $0.975(0.772-1.230)$ & $0.877(0.697-1.103)$ & $0.773(0.609-0.981)$ \\
\hline Reduction in risk \% $(95 \% \mathrm{CI})$ & & $2.5(-23.0-22.8)$ & $12.3(-10.3-30.3)$ & $22.7(1.9-39.1)$ \\
\hline \multicolumn{5}{|l|}{ ICS/LABD } \\
\hline Subjects & 1102 & 1164 & 1157 & 1194 \\
\hline \multicolumn{5}{|l|}{ Active versus placebo } \\
\hline Hazard ratio $(95 \% \mathrm{CI})$ & & $1.006(0.877-1.154)$ & $0.987(0.861-1.132)$ & $0.766(0.665-0.882)$ \\
\hline Reduction in risk \% $(95 \% \mathrm{CI})$ & & $-0.6(-15.4-12.3)$ & $1.3(-13.2-13.9)$ & $23.4(11.8-33.5)$ \\
\hline
\end{tabular}

patients. However, although prior exacerbation history will affect risk of future exacerbations, there is little to indicate that the relative reduction of exacerbations by medical treatment varies according to exacerbation frequency. Treatment prior to study entry will also depend on where the study is carried out as treatments vary significantly across the globe, as this study has also found. This could be one of the
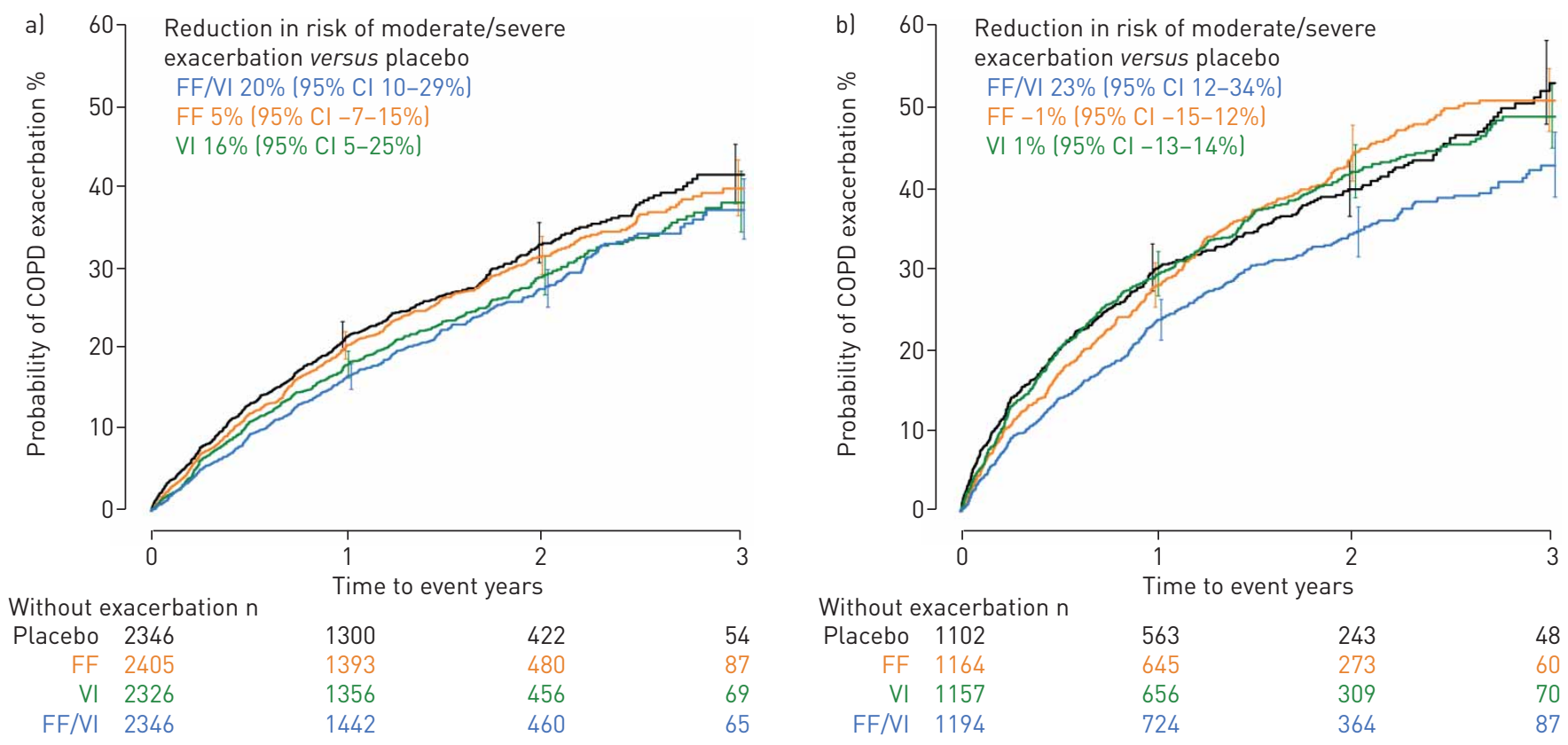

FIGURE 2 Risk of moderate/severe exacerbations in the four treatment groups (placebo, fluticasone furoate (FF), vilanterol (VI) and FF/VI) according to previous treatment use. a) Those receiving no inhaled pre-treatment maintenance medication; b) those on inhaled corticosteroids and long-acting bronchodilators as previous treatment use. COPD: chronic obstructive pulmonary disease. 
reasons for studies in different regions showing different treatment effects, as previously seen for ICS/ LABA and health status [10], and most recently for tiotropium on lung function decline [11, 12].

In a placebo-controlled trial like SUMMIT, there will be patients who have their prior treatment withdrawn. Recent publications have suggested that careful withdrawal of ICS is well tolerated and not associated with exacerbation risk or any substantial loss of lung function if patients continue to take LABD [13]. However, a number of prior studies have suggested that some patients with COPD do deteriorate after stopping ICS [14, 15], including some with mild disease [16], and similar effects have been described for theophylline [17]. In addition, the same discussion about pre-enrolment medication has come up for a study comparing a fixed triple combination inhaler with an ICS/LABA [18, 19]. No studies have adequately examined the withdrawal of LABD or LABD/ICS on patient outcomes despite the potential implications for both clinical practice and the design of clinical trials. In SUMMIT, we chose a lower limit of FEV 1 of $50 \%$ predicted in the inclusion criteria as we believed no study had shown beneficial effects of treatments that would preclude a placebo arm in this patient group. However, COPD is a heterogeneous disease with variable need for, and response to, therapy and caution should be taken when withdrawing treatments in patients in whom a physician has found more intense treatment to be indicated. Our study indicated that the effect of FF/VI on mortality is larger in those previously on an ICS/LABD than in those who were treatment naïve. However, we have made multiple comparisons in these analyses and the overall p-value of 0.440 for the mortality analysis cautions us not to overinterpret these mortality findings. In addition, the effect of those previously on LABD was also larger and no clear differential effect was seen on exacerbations. The SUMMIT trial has been criticised for withdrawing long-acting anticholinergics [20] but given that only $15 \%$ were on this drug before inclusion [2] and tiotropium could be used during the trial at the investigator's discretion, we cannot evaluate this in our analyses.

Regarding the safety aspect, our analyses showed that this type of post hoc analysis is not straightforward. We are used to analysing randomised trials on the premise that external factors are well balanced. However, in our event-driven study, there was a significant variation in study exposure when we divided the study population according to prior treatments. This is likely due to different countries being included in the study at different time points, most notably with longer study duration for patients in the USA and Western Europe. This also means that differences in prior treatment are likely to be linked to differences in healthcare, factors that are not registered and not accounted for in the analyses.

A strength of our study is its size and the presence of a placebo arm. However, it is limited to patients with moderate COPD and it is possible that this affects our findings. Yet in the TORCH study, where most patients had severe or very severe disease, previous treatment use did not affect treatment effects within the trial, indicating that COPD severity may not be a confounder. Our study also contains only patients with both COPD and cardiovascular comorbidity, but it is not immediately obvious why our findings would not be relevant for patients with either no comorbidities or additional comorbidities.

In conclusion, our findings confirm previous analyses of the TORCH trial showing no consistent effects of previous treatment use on exacerbations in a randomised controlled trial of FF, VI or FF/VI combined. However, patients withdrawn from an ICS/LABA appear to show better effects of an active treatment compared to placebo with regard to mortality. These presented analyses, however, are all post hoc subgroup analyses that should be interpreted with caution.

Conflict of interest: J. Vestbo reports that he was reimbursed for his work as chair of the SUMMIT Steering Committee by GlaxoSmithKline during the conduct of the study; and that he has received consultancy fees for the COPD Phase 2 and 3 programme and payment for lectures including service in a speaker bureau from GlaxoSmithKline, Chiesi Pharmaceuticals, Boehringer Ingelheim, Novartis and AstraZeneca, outside the submitted work. M. Dransfield reports consulting fees from GlaxoSmithKline during the conduct of the study; and grants from the Department of Defense and the NIH, personal fees for consulting and contract clinical trials from Boehringer Ingelheim, GlaxoSmithKline, AstraZeneca, PneumRx/BTG and Boston Scientific, support for contract clinical trials from Novartis, Yungiin and Pulmonx, and consulting fees from Genentech and Quark Pharmaceuticals, outside the submitted work. J.A. Anderson is an employee of and holds shares in GlaxoSmithKline. R.D. Brook reports work on the SUMMIT Steering Committee for GSK during the conduct of the study. P.M.A. Calverley reports personal fees from Astra Zeneca Pharmaceuticals, GSK, Boehringer Ingelheim and Recipharm, outside the submitted work. B.R. Celli reports support for a research site from AstraZeneca, and consulting fees from GlaxoSmithKline, Boehringer Ingelheim, Novartis, Chiesi and Menarini, all outside the submitted work. N.J. Cowans is an employee of Veramed Limited, a company that GSK paid to carry out the statistical analysis for this article. C. Crim reports is an employee of and holds restricted shares in GlaxoSmithKline. F. Martinez reports that GSK was the supporter of the parent study to the current study; and honoraria and nonfinancial travel support from the American College of Chest Physicians for COPD CME programmes in India, personal fees and nonfinancial travel support from AstraZeneca for COPD advisory boards, the study steering committee and an ALAT presentation, personal fees and nonfinancial support from Boehringer Ingelheim for a COPD advisory board, an ATS presentation, an IPF study steering committee, an IPF disease state presentation at ALAT and a progressive pulmonary fibrosis DSMB, nonfinancial support from ProterrixBio for a COPD scientific advisory board, honoraria from Columbia University for a COPD CME programme, honoraria and nonpersonal travel support from ConCert for a COPD advisory board, personal fees and nonpersonal travel support from Genentech for a COPD 
advisory board, personal fees and nonfinancial support from GlaxoSmithKline for COPD advisory boards, a study steering committee, a DSMB and an ERS presentation, honoraria from Haymarket Communications for a COPD CME presentation, honoraria and nonpersonal travel support from the Inova Fairfax Health System for a COPD CME presentation, honoraria from Integritas for a COPD CME presentation, honoraria and nonpersonal travel support from MD Magazine for a COPD CME programme, honoraria from Methodist Hospital Brooklyn for a COPD CME programme, honoraria and nonpersonal travel support from Miller Communications for COPD and IPF CME programmes, honoraria and nonpersonal travel support from the National Association for Continuing Education for COPD and IPF CME programmes, honoraria and nonpersonal travel support from Novartis for a COPD advisory board and international meeting COPD presentations, honoraria from New York University for a COPD CME programme, personal fees and nonfinancial support from Pearl Pharmaceuticals for COPD advisory boards and a COPD steering committee, honoraria and nonpersonal travel support from PeerView Communications for COPD and IPF CME programmes, honoraria and nonpersonal travel support from Prime Communications for COPD CME programmes, honoraria and nonpersonal travel support from the Puerto Rican Respiratory Society for a COPD and IPF CME programme, honoraria and nonpersonal travel support from Chiesi for a COPD CME presentation and an advisory board, honoraria and nonpersonal travel support from Sunovion for COPD advisory boards, honoraria and nonpersonal travel support from Theravance for a COPD advisory board, honoraria from Unity for COPD teleconferences, honoraria from UpToDate for COPD CME, honoraria from WebMD/MedScape for COPD CME presentations, honoraria from the Western Connecticut Health Network for COPD CME presentations, coauthorship from Afferent/Merck for an IPF study steering committee, honoraria from Academic CME for IPF CME programmes, coauthorship from Gilead for an IPF study steering committee, nonfinancial support from Nitto for an IPF study teleconference and steering committee, honoraria from Patara for venture capital expert advice for an IPF study, honoraria from PeerView for an IPF CME presentation, honoraria from PlatformIQ for an IPF CME programme, honoraria and nonpersonal travel support from Potomac for IPF CME presentations, nonfinancial support from Stromedix/Biogen for an IPF study DSMB and IPF study steering committee, honoraria and nonpersonal travel support from the University of Alabama Birmingham for and IPF CME presentation, coauthorship from Veracyte for an IPF study steering committee, nonpersonal travel support to attend an IPF study meeting and an honorarium for an advisory board from Zambon, an honorarium for acting as AJRCCM DE from the American Thoracic Society, grants from the NIH, an honorarium and travel support from the Physicians Education Resource for a COPD CME programme, an honorarium from Rockpointe for a COPD CME programme, nonfinancial support from Prometic for an IPF steering committee, grants from Rare Disease Health are Communications, and an honorarium and travel support from Teva for a COPD advisory board, outside the submitted work. D.E. Newby reports consultancy fees from GSK for serving on the trial steering committee during the conduct of the study, and grants and personal fees for other research and consultancy activities from GSK, outside the submitted work. J. Yates is an employee of and holds shares in GSK. P. Lange reports personal fees for membership of the data monitoring board from GSK during the conduct of the study; and consultancy and teaching fees from AstraZeneca, Boehringer Ingelheim, Chiesi and GSK, outside the submitted work.

Support statement: The SUMMIT trial was sponsored by GlaxoSmithKline. J. Vestbo was supported by the National Institute of Health Research Manchester Biomedical Research Centre. Funding information for this article has been deposited with the Crossref Funder Registry.

\section{References}

1 Calverley PMA, Anderson JA, Celli B, et al. Salmeterol and fluticasone propionate and survival in chronic obstructive pulmonary disease. N Engl J Med 2007; 356: 775-789.

2 Vestbo J, Anderson JA, Brook RD, et al. Fluticasone furoate and vilanterol and survival in chronic obstructive pulmonary disease with heightened cardiovascular risk (SUMMIT): a double-blind randomised controlled trial. Lancet 2016; 387: 1817-1826.

3 Tashkin DP, Celli B, Senn S, et al. A 4-year trial of tiotropium in chronic obstructive pulmonary disease. $N$ Engl J Med 2008; 359: 1543-1554.

4 Dransfield MT, Bourbeau J, Jones PW, et al. Once-daily inhaled fluticasone furoate and vilanterol versus vilanterol only for prevention of exacerbations of COPD: two replicate double-blind, parallel-group, randomised controlled trials. Lancet Respir Med 2013; 1: 210-223.

5 Suissa S, Ernst P, Vandemheen KL, et al. Methodological issues in therapeutic trials of COPD. Eur Respir J 2008; 31: 927-933.

6 Ernst P, Saad N, Suissa S. Inhaled corticosteroids in COPD: the clinical evidence. Eur Respir I 2015; 45: 525-537.

7 Keene ON, Vestbo J, Anderson JA, et al. Methods for therapeutic trials in COPD: Lessons from the TORCH trial. Eur Respir J 2009; 34: 1018-1023.

8 Andreas S, Janson C, van den Berge M, et al. Cardiac impact of inhaled therapy in the largest randomised placebo-controlled trial in COPD history: have we reached the SUMMIT? ERJ Open Res 2016; 2: 00055-02016.

9 Calverley PM, Tetzlaff K, Dusser D, et al. Determinants of exacerbation risk in patients with COPD in the TIOSPIR study. Int J Chron Obstruct Pulmon Dis 2017; 12: 3391-3405.

10 Jones PW, Anderson JA, Calverley PMA, et al. Health status in the TORCH study of COPD: treatment efficacy and other determinants of change. Respir Res 2011; 12.

11 Zhou Y, Zhong NS, Li X, et al. Tiotropium in early-stage chronic obstructive pulmonary disease. N Engl J Med 2017; 377: 923-935.

12 Decramer M, Celli B, Kesten S, et al. Effect of tiotropium on outcomes in patients with moderate chronic obstructive pulmonary disease (UPLIFT): a prespecified subgroup analysis of a randomised controlled trial. Lancet 2009; 374: 1171-1178.

13 Magnussen H, Disse B, Rodriguez-Roisin R, et al. Withdrawal of inhaled glucocorticoids and exacerbations of COPD. N Engl J Med 2014; 371: 1285-1294.

14 Wouters EF, Postma DS, Fokkens B, et al. Withdrawal of fluticasone propionate from combined salmeterol/ fluticasone treatment in patients with COPD causes immediate and sustained disease deterioration: a randomised controlled trial. Thorax 2005; 60: 480-487. 
15 Kunz LI, Postma DS, Klooster K, et al. Relapse in FEV1 decline after steroid withdrawal in COPD. Chest 2015; 148: 389-396.

16 Choudhury AB, Dawson CM, Kilvington HE, et al. Withdrawal of inhaled corticosteroids in people with COPD in primary care: a randomised controlled trial. Respir Res 2007; 8: 93

17 Kirsten DK, Wegner RE, Jörres RA, et al. Effects of theophylline withdrawal in severe chronic obstructive pulmonary disease. Chest 1993; 104: 1101-1107.

18 Suissa S, Ariel A. The FULFIL trial. Am J Respir Crit Care Med 2018; 197: 542.

19 Lipson DA, Barnacle H, Birk R, et al. Reply to Suissa and Ariel. Am J Respir Crit Care Med 2018; 197: 542-543.

20 Suissa S. Will SUMMIT reach the peak in COPD? Thorax 2014; 69: 405-407. 